\title{
Transformation of defects in semiconductor structures under the influence of microwave electromagnetic radiation, which is stimulated by drift phenomena
}

\author{
G.V. Milenin ${ }^{1}$, R.A. Redko ${ }^{1,2}$ \\ ${ }^{1}$ V. Lashkaryov Institute of Semiconductor Physics, National Academy of Sciences of Ukraine, \\ 41, prospect Nauky, 03680 Kyiv, Ukraine \\ ${ }^{2}$ State University of Telecommunications, 7, Solomenska str., 03680 Kyiv, Ukraine \\ E-mail:milenin.gv@gmail.com; redko.rom@gmail.com
}

\begin{abstract}
The mechanisms of directional motion of highly mobile charged point defects in semiconductor structures under the non-thermal action of microwave radiation have been considered. The effects of particle drift along the direction of the electric field of a homogeneous electromagnetic wave and in the direction of its propagation, as well as the appearance of a gradient ponderomotive force in an inhomogeneous wave have been analyzed. The features of the appearance of an electric force acting on charged point defects as a result of formation of electron-hole junctions around charged dislocations have been studied. Analytical relationships describing the dynamics of impurity ions in semiconductor structures exposed to microwave radiation have been presented.
\end{abstract}

Keywords: microwave radiation, charged defect, particle drift, ponderomotive force.

https://doi.org/10.15407/spqeo23.01.46

PACS 72.30.+q, 72.90.+y

Manuscript received 10.12.19; revised version received 22.01.20; accepted for publication 18.03.19; published online 23.03.19.

\section{Introduction}

In [1-4], the mechanisms of transformation of charged defects in semiconductor structures under the nonthermal action of electromagnetic radiation of the microwave range, which were based on resonant (vibrational) phenomena, were discussed. Their essence was the coincidence of frequency inherent to this radiation with the intrinsic and ion-plasma frequencies of vibrations typical to dislocations and clusters of impurity-defect complexes. At a resonant frequency, due to a sharp increase in the amplitude of oscillations, dislocations detach and subsequently move under the influence of a mosaic of internal mechanical stresses of the crystal, as well as impurity-defect complexes decay and diffusion of decay products occur. At the same time, it should be noted that in addition to the above, other mechanisms providing evolution of defects under the influence of microwave radiation are not excluded. They are based on the idea that in a microwave electromagnetic field, a charged defect not only oscillates, but also drifts. For example, in $[5,6]$, the ponderomotive effect arising as a result of nonlinear interaction of the electric component of microwave electromagnetic field with charged point defects in the near-surface region of ionic crystals was experimentally investigated and theoretically substantiated.

This article is devoted to studying the drift mechanisms for highly mobile charged point defects in semiconductor crystals under the influence of microwave radiation.

2. Drift of charged point defects inside semiconductor structures exposed to homogeneous microwave electromagnetic field

During propagation of a microwave electromagnetic wave inside a semiconductor crystal, its attenuation is observed. If the latter can be neglected, then the microwave field in the crystal can be considered as homogeneous. Otherwise, if extinction cannot be neglected, the corresponding microwave electromagnetic field should be considered as inhomogeneous. Let us first analyze the features of the drift of charged point defects in a homogeneous microwave field. 
In $[7,8]$, it was shown that a free charged particle with the mass $m$ and charge $e$ in an electromagnetic field with an electric component $E_{0} \exp (i \omega t)$ not only oscillates but also performs a systematic drift in parallel to the direction of this electric field. The velocity of this directional movement $v_{1}$ can be defined as in [7]:

$$
v_{1}=-\frac{e E_{0} \sin \varphi}{m \omega}
$$

where $E_{0}$ is the amplitude of the electric field, $\varphi$ - phase of the field at the initial time, $\omega$ - circular frequency of the field.

As it follows from (1), systematic motion is absent only at $\sin \varphi=0$, and for all other values of the phase $\varphi$, the charged particle systematically moves along the electric field, and depending on the values $\varphi$, the particle drifts in mutually opposite directions [7, 8].

It is quite natural that for an arbitrary phase value $\varphi$ over a finite period of time $t$, the particle will travel over the distance $l_{1}$ equal to:

$$
l_{1}=-\frac{e E_{0} \sin \varphi}{m \omega} t
$$

It should be noted that for the entire set of charged particles, the initial phase $\varphi$ is a random value that obeys a continuous uniform distribution. In turn, since all values of the initial phase are equally probable, the drift velocity averaged over $\varphi$ is equal to zero [7]. It means that, along with the systematic drift of individual charged particles as a whole, directional movement of the entire set of particles is absent.

In addition to drift along the electric vector of the wave, the particle systematically drifts in the direction of wave propagation with the velocity $v_{2}[8,9]$ :

$v_{2}=\frac{e^{2} E_{0}^{2}(\cos 2 \varphi+2)}{4 m^{2} \omega^{2} c}$,

where $c$ is the speed of light.

Accordingly, the expression for the distance $l_{2}$, over which the charged particle moves due to action of electromagnetic radiation, has the form:

$$
l_{2}=\frac{e^{2} E_{0}^{2}(\cos 2 \varphi+2)}{4 m^{2} \omega^{2} c} t
$$

Therefore, the charged particle in the field of electromagnetic wave drifts both in the direction of the electric field and in the direction of the wave vector of the latter, the sign of the drift velocity in the direction of wave propagation is always the same, and its value depends on the initial phase [8].

In the expressions (1) to (4), the mass of free charged particles defines as $m$. However, impurity ions in semiconductor crystals are not free, since they have to overcome potential barriers with height $E_{a}$ when moving. This feature of motion of charged point defects can be taken into account, if, in the indicated expressions, by the mass of a singly charged particle we mean some effective mass of an impurity ion:

$m_{\text {eff }}=m \exp \left(E_{a} / k T\right)$

where $m$ is the mass of free ion, $k$ - Boltzmann constant, $T$ - absolute temperature.

\section{Drift of charged point defects inside the semiconductor structure exposed to the inhomogeneous microwave electromagnetic field}

The Gaponov-Miller force, which is proportional to the gradient from the square of the absolute electric field value at a given point, acts on a free charged particle in an inhomogeneous microwave field $E(x, y, z) \exp (i \omega t)$ $[8,10]$ :

$\vec{F}=-\frac{e^{2}}{4 m \omega^{2}} \operatorname{grad}|E(x, y, z)|^{2}$.

We assume that the electric field of an electromagnetic wave has only one component along the axis $y$ perpendicular to the direction of propagation along the axis $x$. Then the wave is homogeneous along the axis $y$ and inhomogeneous along the axis $x$. In this case, for the value of the ponderomotive force (6) in any point $x$ we have:

$F(x)=-\frac{e^{2}}{4 m \omega^{2}} \frac{\partial|E(x)|^{2}}{\partial x}$.

When the electromagnetic microwave radiation acts on the semiconductor structure, the inhomogeneity of the wave occurs due to attenuation, since it propagates in the crystal. The law of variation of the amplitude along the propagation axis has the form [11]:

$E(x)=E_{0} \exp (-\beta x)$,

where $E_{0}$ is the amplitude of oscillations of the electric component of the electromagnetic wave on the surface of semiconductor, $\beta$ is the extinction coefficient of the electromagnetic wave. Then the expression for the force acting on a singly charged point defect is written in the form:

$$
F(x)=\frac{e^{2} \beta E_{0}^{2}}{2 m_{e f f} \omega^{2}} \exp (-2 \beta x) .
$$

The dynamics of behavior inherent to a charged point defect is described by the following equation: 
$m_{e f f} \frac{d^{2} x}{d t^{2}}=F(x)$.

Let us represent the acceleration in the following way:

$\frac{d^{2} x}{d t^{2}}=\frac{d v_{3}}{d t}=\frac{d v_{3}}{d x} \frac{d x}{d t}=v_{3} \frac{d v_{3}}{d x}$

Then for (9), we have:

$m_{\text {eff }} v_{3} \frac{d v_{3}}{d x}=F(x)$

Integrating (11) with account of (8) within the ranges $[0, x]$ and $\left[0, v_{3}\right]$, we obtain that the drift velocity of impurity ions equals:

$v_{3}=\frac{e E_{0}}{\sqrt{2} m_{e f f} \omega}[1-\exp (-2 \beta x)]^{1 / 2}$.

Based on (10) and (12), we find that the change in the velocity of the impurity ion in time obeys the equation:

$\frac{d v_{3}}{v^{2}-v_{0}^{2}}=-\beta d t$

where $v_{0}=e E_{0} / \sqrt{2} m_{\text {eff }} \omega$.

Integrating (13), we obtain:

$v_{3}=v_{0} \frac{1-\exp \left(-2 \beta v_{0} t\right)}{1+\exp \left(-2 \beta v_{0} t\right)}$

Let us consider the case of short durations of exposure to microwave radiation (non-thermal effect) on the semiconductor structure, at which $2 \beta v_{0} t<<1$. Taking into account that under this exposure $\exp \left(-2 \beta v_{0} t\right)=1-2 \beta v_{0} t$, for $v_{3}$ we have:

$v_{3}=\frac{e^{2} E_{0}^{2} \beta t}{2 m_{e f f}^{2} \omega^{2}}$.

Therefore, an impurity ion, the initial velocity of which is zero, under the influence of microwave radiation moving uniformly accelerated, over time $t$ will move the distance $l_{3}$ :

$l_{3}=\int_{0}^{t} v_{3} d t=\frac{e^{2} E_{0}^{2} \beta t^{2}}{4 m_{e f f}^{2} \omega^{2}}$.
When using (15) and (16) for calculations, it is necessary to have an idea of the parameter $\beta$. The extinction coefficient $\beta$ depends on the prevailing mechanism of electromagnetic radiation absorption inside semiconductor crystals. In the microwave range, absorption is caused by free charge carriers. If in the microwave frequency range the condition $\omega \tau<<1$ is satisfied (which takes place in experiments on the influence of microwave processing on semiconductor structures), then the corresponding expression for the absorption coefficient by free charge carriers $\alpha$ has the form [11]:

$\alpha=\frac{\sigma}{c \varepsilon_{0} \bar{n}}$

where $\tau=m_{n} \mu_{n} / e$ is the relaxation time, $\sigma=e n \mu_{n}-$ specific electrical conductivity of semiconductor, $m_{n}-$ effective mass of electrons in a crystal, $\mu_{n}$ - electron mobility, $n$ - concentration of free electrons in a semiconductor crystal, $\bar{n}$ - real part of the complex refraction index, $\varepsilon_{0}-$ electric constant of vacuum.

The coefficients $\beta$ and $\alpha$ are related by the relation [11]:

$\beta=\frac{\alpha}{2}$

Using (17) involves knowledge of the numerical value $\bar{n}$. It is known that the real $\bar{n}$ and imaginary $\chi$ parts of the complex refractive index are determined by the expressions [11]:

$\bar{n}^{2}=\chi^{2}+\varepsilon$,

$\chi=\frac{\sigma}{2 \bar{n} \varepsilon_{0} \omega}$,

where $\varepsilon$ is the permittivity of a semiconductor crystal.

As a result of solving the system of equations (19) and (20) with respect to $\bar{n}$, we have:

$$
\bar{n}=\sqrt{\frac{\varepsilon}{2}\left[1+\sqrt{1+\left(\frac{\sigma}{\varepsilon_{0} \varepsilon \omega}\right)^{2}}\right]} .
$$

Substituting (21) into (17) and taking into account (18), one can obtain the values of $\beta$.

Let us consider two practically important particular cases. If $\left(\sigma / \varepsilon_{0} \varepsilon \omega\right)>>1$, then

$\bar{n}=\left(\frac{\sigma}{2 \varepsilon_{0} \omega}\right)^{1 / 2}$. 
Then, in accordance with (17) and (18),

$\beta=\frac{1}{c}\left(\frac{\sigma \omega}{2 \varepsilon_{0}}\right)^{1 / 2}$,

that is $\beta^{-1}=\delta_{s}$, where $\delta_{s}=c\left(2 \varepsilon_{0} / \sigma \omega\right)^{1 / 2}$ is the thickness of the skin layer [12]. If $\left(\sigma / \varepsilon_{0} \varepsilon \omega\right)<<1$, then

$\bar{n}=(\varepsilon)^{1 / 2}$

and

$\beta=\frac{\sigma}{2 c \varepsilon_{0} \varepsilon^{1 / 2}}$.

In conclusion, we formulate a criterion for weak and strong attenuation of electromagnetic waves incident onto a semiconductor structure (characterized by homogeneity or inhomogeneity of a microwave field in a crystal). Let us assume that for the thickness $d$ of the semiconductor crystal and the extinction coefficient $\beta$, the following condition is satisfied: $d<<\beta^{-1}$, i.e., attenuation is absent. Otherwise, attenuation becomes significant, and when $d \geq \beta^{-1}$ there is a strong attenuation of electromagnetic waves.

\section{The effect of charged dislocations on the drift of charged point defects in semiconductor structures under action of microwave radiation}

Dangling bonds in the nuclei of edge dislocations can act as acceptors or donors of electrons [13]. If free electron is captured by a dangling bond, then the dislocation becomes negatively charged. Such a negatively charged line repulses free electrons and induces formation of a tube of a positive charge around the dislocation [13]. For sufficiently large values of the charge of dislocation in an $n$-type semiconductor, around this dislocation formation of an inversion layer becomes possible [13] and, consequently, formation of a $p$ - $n$ junction. The effect of rectification of currents due to the formation of an electron-hole junction around a dislocation was experimentally observed in [14]. It was noted that the current-voltage characteristic of a dislocation diode in $n$-type silicon is non-linear and asymmetric, moreover, direct and reverse currents are different by hundred times [14]. The $p-n$ junction thus formed during oscillations of one of the half-waves of alternating electric field of the electromagnetic wave is switched on in the forward direction, and in the opposite direction - during the other. In the closed state, in the dislocation diode the width of the space charge region of the $p-n$ junction increases with increasing voltage, and ionic impurities localized near the dislocations fall into this area. Under the influence of the built-in electric field, highly mobile charged point defects gain the ability to move.
In particular, for a symmetrical stepwise $p$ - $n$ junction in the state of thermal equilibrium, the width of the depletion area $W$ is [15]:

$W=\left(\frac{4 \varepsilon_{0} \varepsilon \varphi}{N}\right)^{1 / 2}$,

in which the following relation takes place:

$\varphi=\frac{2 k T}{e} \ln \frac{N}{n_{i}}$,

where $\varphi$ is the contact potential difference, $N-$ concentration of impurities in the $n$ - and $p$-areas of the junction (it is assumed that all donors and acceptors are ionized), $n_{i}$ - intrinsic concentration of carriers.

For this junction, the maximum width of the depletion region $W_{\max }$ under the action of microwave radiation can be found by solving the equation:

$W_{\max }=\left[\frac{4 \varepsilon_{0} \varepsilon\left(\varphi+E_{0} W_{\max }\right)}{N}\right]^{1 / 2}$,

and it is:

$W_{\max }=\frac{2 \varepsilon_{0} \varepsilon E_{0}}{e N}+\left(\frac{4 \varepsilon_{0}^{2} \varepsilon^{2} E_{0}^{2}}{e^{2} N^{2}}+\frac{4 \varepsilon_{0} \varepsilon \varphi}{e N}\right)^{1 / 2}$.

Let it be: $\bar{E}$ is the average (over the period of oscillations) value of the electric component of electromagnetic wave corresponding to being of the dislocation diode in the closed state and is equal to:

$\bar{E}=\frac{1}{2 \pi} \int_{0}^{\pi} E_{0} \sin \omega t d(\omega t)=\frac{E_{0}}{\pi}$.

Then, on average during a period of electromagnetic oscillations, a force acts on a singly charged impurity ion in the space charge region of the $p$ - $n$ junction:

$$
\bar{F}=e\left(\bar{E}+\frac{\varphi}{\bar{W}}\right)
$$

where $\bar{W}$ is determined from (29) with replacing $E_{0}$ by $\bar{E}$.

The average displacement of a point defect with a zero initial velocity under action of a given force during the period of microwave radiation presence is:

$$
\overline{l_{4}}=\frac{\bar{F}}{2 m_{\text {eff }}} t^{2}=\frac{e\left[\left(E_{0} / \pi\right)+(\varphi / \bar{W})\right]}{2 m_{\text {eff }}} t^{2},
$$

at that $\overline{l_{4}} \leq \bar{W}$. 


\section{Calculation of parameters describing the drift of defects in semiconductor structures under action of microwave radiation}

Let us estimate the values of drift parameters $v_{1}$ and $l_{1}$ for rapidly diffusing singly-charged copper ions in epitaxial semiconductor structures under the influence of a homogeneous microwave field. Let the frequency of electromagnetic radiation is $2.45 \cdot 10^{9} \mathrm{~Hz}$, and $E_{0}=1.5 \cdot 10^{3} \mathrm{~V} / \mathrm{m}$. Taking into account that the mass of free copper ions $m=1.055 \cdot 10^{-25} \mathrm{~kg}$, then at $E_{a}=0.4 \mathrm{eV}$, $\varphi=-\pi / 2, T=300 \mathrm{~K}$ and $t=5 \mathrm{~s}$, the calculation according to formulas (1), (2) and (5) gives the following values for the drift parameters: $v_{1}=0.28 \cdot 10^{-7} \mathrm{~m} / \mathrm{s}$ and $l_{1}=1.4 \cdot 10^{-7} \mathrm{~m}$. At $E_{a}=0.45 \mathrm{eV}$ and unchanged values of the remaining parameters, one can obtain: $v_{1}=0.41 \cdot 10^{-8} \mathrm{~m} / \mathrm{s}$ and $l_{1}=2.1 \cdot 10^{-8} \mathrm{~m}$. As one can see, the drift parameters, ceteris paribus, are characterized by a strong sensitivity to $E_{a}$. Thus, drift effects under action of microwave radiation are most pronounced in relation to defects with high mobility.

\section{Conclusions}

Transformation of the subsystem of highly mobile charged point defects in semiconductor structures during non-thermal microwave processing may be caused by the directional movement of the latter under the influence of an electromagnetic wave. If attenuation of electromagnetic waves incident on a semiconductor crystal can be neglected, then in such a homogeneous microwave field there is a drift of impurity ions both along the direction of the electric field of the wave and in the direction of its wave vector. In the first case, the drift velocity is proportional to the amplitude of oscillations of the electric field and inversely proportional to the frequency of the microwave radiation, as well as to the effective mass of ion. In the second case, these dependences are squared in respect to the corresponding quantities. The consequence of attenuation of the incident electromagnetic waves in the semiconductor structure is that for the corresponding inhomogeneous microwave field, the charged point defects are affected by the Gaponov-Miller force proportional to the gradient of the square of the electric field modulus.

The value of this gradient force is determined by the extinction coefficient of the electromagnetic wave in the semiconductor crystal, the oscillation amplitude of the electric component of the latter on the surface of semiconductor, the radiation frequency and effective mass of the defect.

Impurity ions localized in the vicinity of dislocations under conditions of microwave radiation are subjected to an electric force, the cause of appearance of which is formation of an electron-hole junction around the charged dislocation, in other words, formation of a dislocation diode. This force is proportional to the amplitude of oscillations of the electric field.

\section{References}

1. Ermolovich I.B., Milenin G.V., Milenin V.V., Konakova R.V., Red'ko R.A. Modification of the defect structure in binary semiconductors under action of microwave radiation. Technical Physics. 2007. 52, No 9. P. 1173-1177. https://doi.org/10.1134/S1063784207090113.

2. Milenin G.V., Red'ko R.A. Physical mechanisms and models of the long-term transformations of radiative recombination in $n$-GaAs under microwave irradiation. Semiconductor Physics, Quantum Electronics and Optoelectronics. 2016. 19, No 1. P. 14-22.

https://doi.org/10.15407/spqeo19.01.014.

3. Red'ko R.A., Milenin G.V., Milenin V.V. Mechanisms and possibilities of defect reorganization in III-V compounds due to the nonthermal microwave radiation treatment. Journal of Luminescence. 2017. 192. P. 1295-1299.

https://doi.org/10.1016/j.jlumin.2017.09.013.

4. Milenin G.V., Red'ko R.A. Transformation of structural defects in semiconductors under action of electromagnetic and magnetic fields causing resonant phenomena. Semiconductor Physics, Quantum Electronics and Optoelectronics. 2019. 22, No 1. P. 39-46. https://doi.org/10.15407/spqeo22.01.039.

5. Rybakov K.I., Semenov V.E. Mass transport in ionic crystals induced by the ponderomotive action of a high-frequency electric field. Phys. Rev. B. 1995. 52, No 5. P. 3030-3033. https://doi.org/10.1103/physrevb.52.3030.

6. Rybakov K.I., Semenov V.E., Freeman S.A., Booske J.H., Cooper R.F. Dynamics of microwaveinduced currents in ionic crystals. Phys. Rev. B. 1997. 55, No 6. P. 3559-3567.

https://doi.org/10.1103/PhysRevB.55.3559.

7. Bolotovskii B.M., Serov A.V. Details of the motion of charged nonrelativistic particles in a variable field. Physics-Uspekhi. 1994. 37. P. 515-516. https://doi.org/10.1070/ PU1994v037n05ABEH000111.

8. Bolotovskii B.M., Serov A.V. Special features of motion of particles in an electromagnetic wave. Physics-Uspekhi. 2003. 46. P. 645-655. https://doi.org/ 10.1070/PU2003v046n06ABEH001349.

9. Serov A.V. Distribution of charged particles injected by a point source into a plane electromagnetic wave. Kratkiye soobshcheniya po fizike Fizicheskogo instituta im. P.N. Lebedeva. 2002. No 8. P. 26-32 (in Russian).

10. Gaponov A.V., Miller M.A. Potential wells for charged particles in a high-frequency electromagnetic field. JETP. 1958. 7, No 1. P. 168-169. 
11. Shalimova K.V. Physics of Semiconductors. St.-Peterburg: Publ. House "Lan", 2010 (in Russian).

12. Lifshitz E.M., Pitaevsky L.P. Physical Kinetics, in: Landay L.D., Lifshitz E.M. Theoretical Physics. Vol. X. Moscow, Nauka, 1979 (in Russian).

13. Matare Herbert F. Defect Electronics in Semiconductors. Willey-Intersci., New York, 1971.

14. Mil'shtein S.Kh., Nikitenko V.I. Investigation of local changes of the electric properties of silicon under the influence of individual dislocations. JETP Lett. 1971. 13, No 7. P. 233-235.

15. Sze S.M. Physics of Semiconductor Devices. John Willey \& Sons. New York, 1981.

\section{Authors and CV}

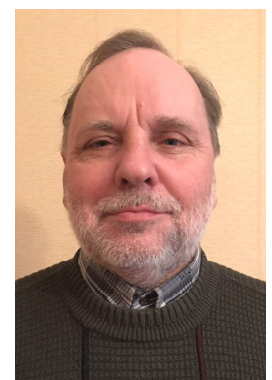

Milenin G.V. $\mathrm{PhD}$ "Solid-State Physics", Senior Researcher at Laboratory of Physical and Technological Problems of Solid-State Microwave Electronics at the V. Lashkaryov Institute of Semiconductor Physics, NAS of Ukraine. Authored more than 80 scientific publications and 5 patents. The areas of scientific interests are semiconductor physics and solidstate electronics.

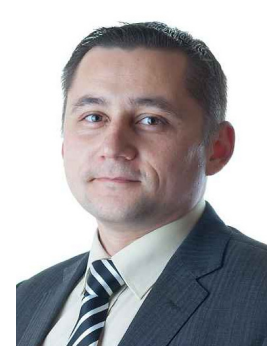

Redko R.A. PhD "Solid-State Physics", Associate Professor, Senior Researcher at Laboratory of Physical and Technological Problems of SolidState Microwave Electronics at the V. Lashkaryov Institute of Semiconductor Physics, NAS of Ukraine. Authored more than 30 scientific publications and 4 patents. The areas of scientific interests are semiconductor physics and solid-state electronics. 